\title{
IMPACTO PRODUCIDO POR LAS CONSTRUCCIONES AGRARIAS SOBRE EL PAISAJE: UNA METODOLOGÍA PARA SU ESTIMACIÓN
}

\author{
(THE VISUAL IMPACT OF AGRICULTURAL BUILDINGS ON LANDSCAPE: A METHOD TO \\ ASSESS IT)
}

Ignacio Cañas Guerrero, Dr. Ingeniero Agrónomo

Escuela Politécnica Superior de Lugo. Univ. de Santiago de Compostela

Francisco Ayuga Tellez, Dr. Ingeniero Agrónomo

E.T.S.I. Agrónomos. U.P.M.

Juan Ortiz Sanz, Ingeniero Agrónomo

Escuela Politécnica Superior de Lugo. Univ. de Santiago de Compostela

Fecha de recepción: 22-IX-95

ESPAÑA

113-35

\section{RESUMEN}

En el presente artículo se expone parte del trabajo realizado para desarrollar un método que permita cuantificar el impacto visual producido por las construcciones agrarias en el paisaje, destinado a aquellos profesionales implicados en su protección. Tras los ensayos realizados, parece confirmarse que el método puede predecir fielmente la opinión del público en cuanto a la calidad del paisaje; que tras un corto periodo de aprendizaje (en torno a diez horas), cualquier técnico es capaz de aplicarlo correctamente; y que permite puntuar el impacto que sobre el paisaje producen éste $y$ otros tipos de instalaciones, permitiendo también predecirlo en la fase de diseño.

\section{SUMMARY}

In this paper it will be exposed part of the work carried out to develop a method to assess the visual impact of agricultural buildings, which is devised for those professionals who are involved in the protection of the lanscape. After the experiments carried out, it seems confirmed that the method can faithfully predict the public opinion about the quality of the landscape; that after a short learning period (about ten hours), it can be correctly applied by any technician; the method also permits the rating if the impact that this and other kinds of buildings will have on the landscape.

\section{Introducción}

Pese a que la legislación española establece la necesidad de proteger el paisaje ante cualquier nuevo proyecto, apenas existe una base metodológica práctica que facilite a los implicados en esta defensa llevar a cabo la misma. Entre estos agentes, se encuentran todos los responsables de la elaboración y ejecución de aquellos proyectos que supongan cambios en el entorno visual, particularmente ingenieros $\mathrm{y}$ arquitectos.
De todas las modificaciones del paisaje, tienen una especial importancia aquellas de carácter puntual y, más en concreto, las producidas por las construcciones agrarias, fundamentalmente por dos razones:

-Habitualmente se deben situar fuera del núcleo rural, es decir, en emplazamientos privilegiados desde el punto de vista visual.

-Su diseño se encuentra fuertemente condicionado a la 
aparición de nuevos materiales (más baratos, pero casi siempre menos estéticos), dado que los promotores de este tipo de edificaciones, por lo general, disponen de márgenes económicos reducidos.

Por este motivo, se está trabajando en el desarrollo de una metodología capaz de identificar y evaluar las posibles fuentes de impactos visuales negativos que producen las construcciones agrarias. En el artículo que se presenta a continuación, se muestra una parte del proceso de elaboración de dicho método, que servirá de herramienta útil a los profesionales de la ingenieríarural y la construcción, responsables de la conservación y mejora de los recursos visuales del medio natural.

\section{Antecedentes}

El trabajo comenzó con el estudio detallado del tratamiento dadoal paisaje por diferentes autores que habían estudiado su valoración (1). Los enfoques dados por estos autores se pueden clasificar en cuatro grandes grupos:

-Enfoques basados en las sensaciones experimentadas por el observador (2) (3).

-Enfoques basados en la posesión de atributos (4) (5) (6) (7) (8).

-Enfoques basados en el "impacto psicológico" que produce un paisaje en el observador (9) (10) (11)

-Enfoques basado en las características de los observadores (12).

Asimismo, se estudiaron los diferentes métodos de valoración del paisaje existentes, y en particular los desarrollados en los Estados Unidos $(13)(14)(15)$. Tras su análisis detallado se vio que éstos, en relación a nuestro propósito, presentaban las siguientes limitaciones:

-Suelen ser bastante generales, al estar diseñados para vastas extensiones.

-Algunos son muy específicos de ciertos tipos de paisaje (montañoso, con vegetación diseminada, etc.)

-Están pensados para expertos de algunas agencias, por lo que su uso no está muy extendido entre los ingenieros y arquitectos

-La escala de valores de la población americana no tiene por qué coincidir con la de nuestra población.

-Hacen un uso muy reducido de descriptores psicológicos y artísticos.

Una vez determinados los posibles inconvenientes de los métodos utilizados por las agencias americanas, se optó por diseñar uno similar a aquéllos, pero más completo y adecuado a los objetivos perseguidos.

Para ello fue preciso realizar una serie de estudios previos, entre ellos el análisis del tratamiento dado a los condicionantes estéticos en la normativa existente (16) y el examen de las principales variables que inciden en la integración de las construcciones agrarias en el paisaje (17).

\section{Descriptores, variables y parámetros}

En el método propuesto se han incorporado, a través de descriptores, los diferentes enfoques del paisaje. Dentro de cada uno de estos descriptores se incluyen de 1 a 4 variables (Tabla 1). A su vez, estas variables se subdividen en 2 , 3, 4 o 5 parámetros, a cada uno de los cuales se les asigna un valor (Tabla 2). Se han contemplado en total 119 parámetros de las 47 variables incluidas en los 16 descriptores. A pesar del elevado número de parámetros, su utilización es muy sencilla, al almacenarse la información en 3 fíchas de fácil cumplimentación (Ver modelos 1, 2 y 3). Éstas se diseñaron de esta forma para facilitar su uso en el campo, pues es allí donde se deben rellenar.

De este modo, es posible clasificar los elementos que intervienen en la valoración estética del paisaje de forma excluyente, exhaustiva y completa. La puntuación de un determinado paisaje resulta de sumar el valor otorgado a cada una de sus variables (para más información del método ver (18) y (19)).

\section{Evaluación de la metodología propuesta}

Unavez diseñadoel método, debíamos cumplir dos objetivos:

a) Comprobar si la puntuación asignada a cada paisaje mediante el modelo propuesto era un buen estimador de las preferencias dei público.

b) Verificar su operatividad, es decir:

b1.- Comprobar el tiempo invertido por una persona en comprender y ser capaz de aplicar el método sin cometer errores.

b2.-Ver si la valoración de los paisajes, hecha independientemente por varias personas entrenadas, no presentaba diferencias significativas entre ellas.

b3.- Comprobar la validez del método para prever el efecto causado por una construcción en el entorno visual.

El experimento diseñado comprendía una primera fase en la que se comparó el valor otorgado al paisaje por el público y el asignado al mismo por los autores, siguiendo la metodología propuesta. En unasegunda fase, se contrastaron 
las valoraciones hechas por diferentes personas entrenadas en el manejo del método, comparándolas entre si y con la opinión del público.

Para realizar el ensayo, se seleccionaron 96 fotografías de diferentes tipos de paisajes ( $\sin$ modificar, muy degradados, rururbanos, montañosos, costeros, etc.), que se dividieron en dos grupos (Serie Azul y Serie Roja) de 48 imágenes cada uno. Para su manejo, se montaron en cartulinas A4, a razón de 8 fotografias de $9 \times 5,5$ por hoja.
La opinión del público se estudió a través de las valoraciones de las 96 fotografías, mediante encuestas realizadas a 364 personas. Cada una de éstas puntuó entre 1 -Muy malay 5 - Muy buena- las 48 imágenes de una de los dos series.

En cuanto a la aplicación del método por personas diferentes a los autores, se contó para este fin con la colaboración de 5 estudiantes de Ingeniería y 2 Ingenieros Agrónomos. Todos ellos lo estudiaron y utilizaron para puntuar las 48 de la Serie Azul.

TABLA 1

Descripciones y variables del método

\begin{tabular}{|c|c|c|c|c|}
\hline DESCRIPTOR & \multicolumn{4}{|c|}{ VARIABLE } \\
\hline Agua & Tipo & Orillas & Movimiento & Cantidad \\
\hline Forma del terreno & \multicolumn{4}{|c|}{ Tipo } \\
\hline Vegetación & Cubierta & Diversidad & Calidad & Tipo \\
\hline Nieve & \multicolumn{4}{|c|}{ Cubierta } \\
\hline Fauna & Presencia & Interés & \multicolumn{2}{|c|}{ Facilidad de verse } \\
\hline Usos suelo & \multicolumn{2}{|c|}{ Tipo } & \multicolumn{2}{|c|}{ Intensidad pob. } \\
\hline Vistas & \multicolumn{2}{|c|}{ Amplitud } & \multicolumn{2}{|c|}{ Tipo } \\
\hline Sonidos & \multicolumn{2}{|c|}{ Presencia } & \multicolumn{2}{|c|}{ Tipo } \\
\hline Olores & \multicolumn{2}{|c|}{ Presencia } & \multicolumn{2}{|c|}{ Tipo } \\
\hline Recursos culturales & Presencia & Tipo & Fac. de verse & Interés \\
\hline Els. alteran carácter & Intrusión & Fragmentación & Línea horiz. & Tapa vistas \\
\hline Forma & Diversidad & Contraste & \multicolumn{2}{|c|}{ Compatibilidad } \\
\hline Color & Diversidad & Contraste & \multicolumn{2}{|c|}{ Compatibilidad } \\
\hline Textura & Diversidad & Contraste & \multicolumn{2}{|c|}{ Compatibilidad } \\
\hline Unidad & \multicolumn{2}{|c|}{ Líneas estructurales } & \multicolumn{2}{|c|}{ Proporción } \\
\hline Expresión & Afectividad & Estimulación & Simb & mo \\
\hline
\end{tabular}


TABLA 2

Ejemplo de subdivisión y valoración de los parámetros de algunas variables

\begin{tabular}{|c|c|c|c|}
\hline DESCRIPTOR & VARIABLE & PARÁMETRO & VALOR \\
\hline \multirow{8}{*}{ Usos del suelo } & \multirow{5}{*}{ Tipo } & Industrial & 0 \\
\hline & & Minero & 0 \\
\hline & & Urbano & 0 \\
\hline & & Agrícola & A \\
\hline & & Salvaie & 15 \\
\hline & \multirow{3}{*}{$\begin{array}{c}\text { Intensidad } \\
\text { de } \\
\text { población }\end{array}$} & Muy poblado & $A=1$ \\
\hline & & Poblado & $A=5$ \\
\hline & & Poco Poblado & $A=10$ \\
\hline \multirow{8}{*}{ Vistas } & \multirow{5}{*}{ Amplitud } & $<45^{\circ}$ & 0 \\
\hline & & $45^{\circ}-90^{\circ}$ & 0,5 \\
\hline & & $90^{\circ}-180^{\circ}$ & 1 \\
\hline & & $180^{\circ}-270^{\circ}$ & 1,5 \\
\hline & & $>270^{\circ}$ & 2 \\
\hline & \multirow{3}{*}{ Tipo } & Baia $(<1.500 \mathrm{~m})$ & 0 \\
\hline & & Media $(1.500-5.000 \mathrm{~m})$ & 1 \\
\hline & & Panorámica $(>5.000 \mathrm{~m})$ & 3 \\
\hline
\end{tabular}

\begin{tabular}{|c|c|c|c|c|c|}
\hline \multicolumn{3}{|c|}{$\begin{array}{l}\text { INVENTARIO RECURSOS } \\
\text { MONICIPIO: } \\
\text { LOCALIZACION (INBTALACIÓN): } \\
\text { PTO DI OBBSRVACIÓN: } \\
\text { NO: }\end{array}$} & AIs & & $\begin{array}{l}\text { ExchA } 2 \\
\text { HOJA } 1\end{array}$ \\
\hline RECURSOS V & LISUAL & & & & \\
\hline 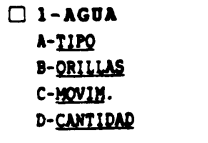 & $\begin{array}{l}\text { 2ONA PANTA } \\
\text { SIN VEGET. } \\
\text { NINGUNO } \\
\text { BNJA }\end{array}$ & $\begin{array}{l}\text { ARROYO } \\
\text { LIGERO }\end{array}$ & $\begin{array}{l}\text { RIO } \\
\text { CON VEGET. } \\
\text { NENDPROS } \\
\text { NEDIA }\end{array}$ & $\begin{array}{l}\text { LAGO/PNITA } \\
\text { RIPIDO }\end{array}$ & $\begin{array}{l}\text { MAR } \\
\text { MUCH. VEG. } \\
\text { CASCADA } \\
\text { ALTA }\end{array}$ \\
\hline $\begin{array}{l}\text { D2-PORMA DEL } \\
\text { A-TIPQ }\end{array}$ & $\begin{array}{l}\text { TRRRENO } \\
\text { LLWNO }\end{array}$ & $\cos \pi \AA$ & COLIMAS & MONTAROSO & \\
\hline $\begin{array}{l}\text { Q 3-VBGETACIÓX } \\
\text { A-CURIBRTA } \\
\text { B-DIVERSID } \\
\text { C-CALIDAD } \\
\text { D-TLPO }\end{array}$ & $\begin{array}{l}\text { SSI } \\
\text { POCA } \\
\text { RECULAR } \\
\text { M. SECANO }\end{array}$ & A. RECADiO & $\begin{array}{l}\text { 25-501 } \\
\text { PRESERTZ } \\
\text { BUEA } \\
\text { ARBOSTIVO }\end{array}$ & PRIDERA & $\begin{array}{l}\text { 775-1004 } \\
\text { BASTANTE } \\
\text { MUY BUDAM } \\
\text { ARBOBREO }\end{array}$ \\
\hline $\begin{array}{r}\text { Q 4-nisve } \\
\text { A-cupisers }\end{array}$ & $<51$ & $5-254$ & 25-501 & $50-754$ & $>75-1004$ \\
\hline 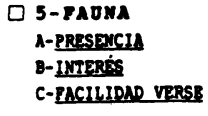 & $\begin{array}{l}\text { PRESENTES } \\
\text { HEDIOCRE } \\
\text { MEDIOCRE }\end{array}$ & & & & $\begin{array}{l}\text { ABUNDANTB } \\
\text { BUENO } \\
\text { BUENA }\end{array}$ \\
\hline $\begin{array}{l}\text { C-U8808 DEL 85 } \\
\text { A-TIPO. } \\
\text { B-INTENSIDAD POR }\end{array}$ & $\begin{array}{l}\text { OELO } \\
\text { INDOSTRIAL } \\
\text { MOY POB. }\end{array}$ & MINERO & $\begin{array}{l}\text { URBNSO } \\
\text { POBLLDO }\end{array}$ & AGRICOL. & $\begin{array}{l}\text { SALVAJE } \\
\text { POCO POBL }\end{array}$ \\
\hline $\begin{array}{c}\text { D 7-VISTAS } \\
\text { A-NAPLITUP }\end{array}$ & ése & 458-90 & $908-1809$ & 1808-2708 & $>2700$ \\
\hline 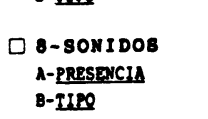 & $\begin{array}{l}\text { PRESETTES } \\
\text { MOLESTOS }\end{array}$ & & INDI FERENTES & & $\begin{array}{l}\text { DOMINANTES } \\
\text { APSONIOSOS }\end{array}$ \\
\hline $\begin{array}{l}\text { D9-OLORES } \\
\text { A-PRESEICIA } \\
\text { B-TIPS }\end{array}$ & $\begin{array}{l}\text { PRESEITES } \\
\text { roLESTOS }\end{array}$ & & INDIFERENTES & & $\begin{array}{l}\text { DOMINANTES } \\
\text { AREONIOSOS }\end{array}$ \\
\hline
\end{tabular}

Modelo 1.- Inventario Recursos Paisaje. Ficha 2. Hoja 1. Descriptores 1 a9.

\begin{tabular}{|c|c|c|c|}
\hline $\begin{array}{l}\text { INUENTARIO } \\
\text { MONICIPIO: } \\
\text { LOCALIZACIOK }\end{array}$ & $\begin{array}{l}\text { O RECURBOB } \\
\text { (IXsTALACIÓN): }\end{array}$ & $\begin{array}{r}\text { PAIBAJE } \\
\text { Pro DI }\end{array}$ & $\begin{array}{l}\text { EICKA? } \\
\text { HOJA } 2 \\
\text { IACION We: }\end{array}$ \\
\hline 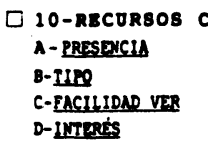 & $\begin{array}{l}\text { COLTORALES } \\
\text { PRESDITES } \\
\text { POPOLRR } \\
\text { MEDIOCRE } \\
\text { MEDIOCRE }\end{array}$ & & $\begin{array}{l}\text { ABUNDANTES } \\
\text { HISTORICO } \\
\text { BUENA } \\
\text { BUENO }\end{array}$ \\
\hline 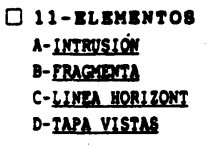 & $\begin{array}{l}\text { OUE ALTERAN EL } \\
\text { BASO } \\
\text { ALCO } \\
\text { ALCO } \\
\text { ALCO }\end{array}$ & $\begin{array}{l}\text { CARACTIRR } \\
\text { MED10 } \\
\text { MEDIO }\end{array}$ & $\begin{array}{l}\text { ALTO } \\
\text { BASTANTE } \\
\text { BLSTANTE } \\
\text { BASTANTE }\end{array}$ \\
\hline \multicolumn{4}{|c|}{ RECURSOS ESTETICOS } \\
\hline 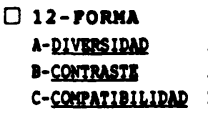 & $\begin{array}{l}\text { Aleoven } \\
\text { AlGorso } \\
\text { no }\end{array}$ & & $\begin{array}{l}\text { DOMINNATRE } \\
\text { DOMINANTE } \\
\text { Si }\end{array}$ \\
\hline 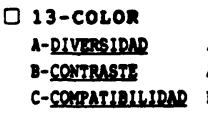 & $\begin{array}{l}\text { Aloon } \\
\text { Alomeno } \\
\text { no }\end{array}$ & & 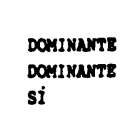 \\
\hline 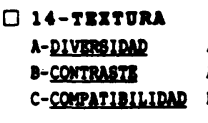 & $\begin{array}{l}\text { Alcova } \\
\text { Alcosso } \\
\text { no }\end{array}$ & & $\begin{array}{l}\text { DOWIMNMTR } \\
\text { DOWINANTE } \\
\text { Si }\end{array}$ \\
\hline 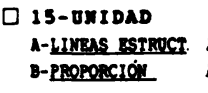 & $\begin{array}{l}\text { ALComa } \\
\text { Aloma }\end{array}$ & & $\begin{array}{l}\text { DOWINNATR } \\
\text { DOMINANTE }\end{array}$ \\
\hline 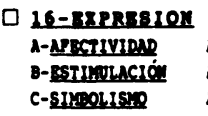 & 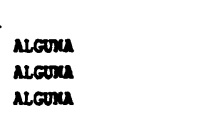 & & $\begin{array}{l}\text { DOMINANTE } \\
\text { DOWINANTE } \\
\text { DOMINANTE }\end{array}$ \\
\hline $\begin{array}{l}\text { O COKEWTARI OS } \\
\text { SI SE CREE CONVE, } \\
\text { OASERVA EN EL, D }\end{array}$ & SO DE LA HOJA. & & \\
\hline
\end{tabular}

ModelQ 2.- Inventario Recursos Paisaje. Ficha 2. Hoja 2. Descriptores 10 a 16.

(c) Consejo Superior de Investigaciones Científicas Licencia Creative Commons 3.0 España (by-nc) 


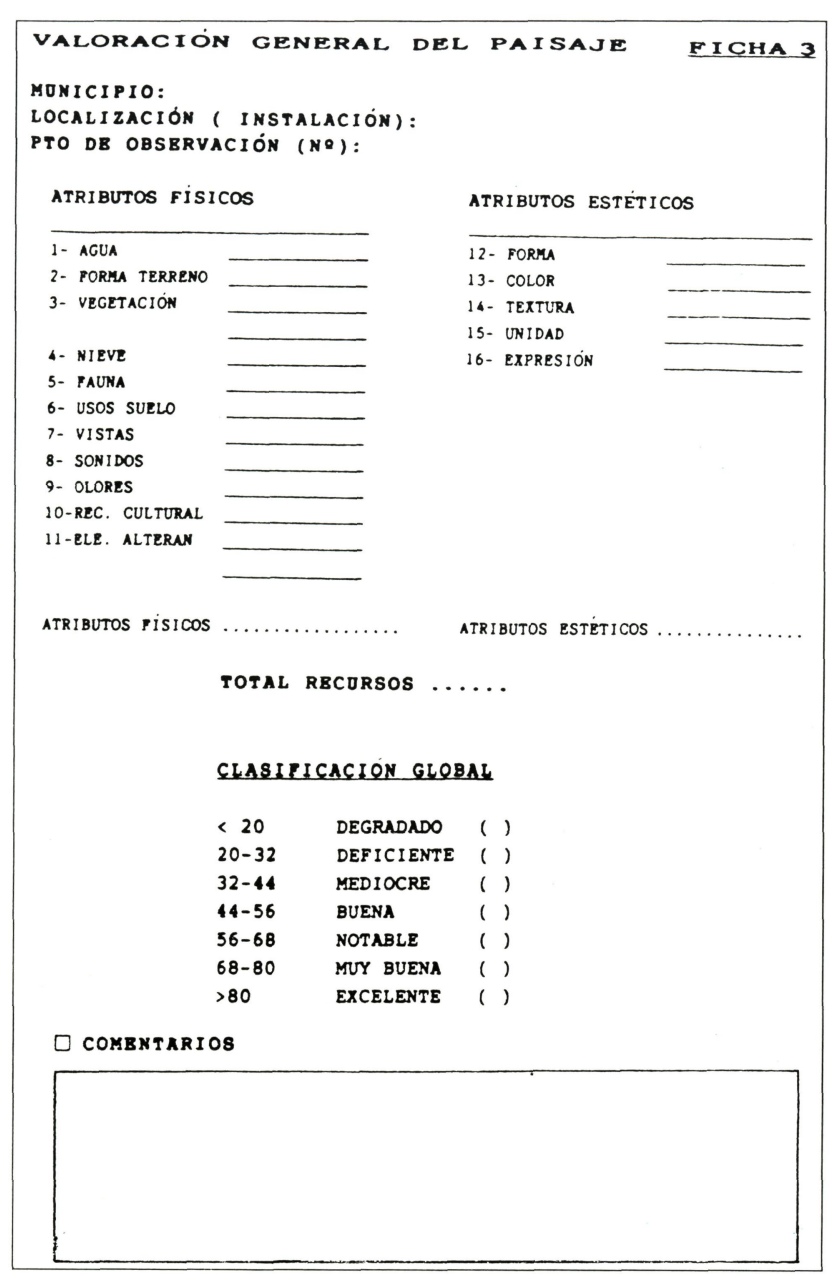

Modelo 3.- Valoración General del Paisaje. Ficha 3. Resumen valoración por atributos. Clasificación global del paisaje y comentarios.

\section{Resultados del ensayo}

Para comprobar la capacidad predictora del modelo, se calculó el coeficiente de correlación $r$ de Pearson entre la media dada por las encuestas a cada fotografía y la que se estimó por el método de evaluación propuesto. El resultado de este cálculo arrojó un índice de correlación $r=0,97$. Además, la pendiente $(\mathrm{b}=1,011)$ y la ordenada en el origen $(\mathrm{a}=-1,3897)$ de la recta ajustada son muy próximas a los valores ideales $(\mathrm{b}=1$ y a $=0)$.).(Gráfico 1$)$.

En cuanto a la operatividad del método, se determinó la media de la puntuación otorgada a cada foto por las 7 personas que las evaluaron. De nuevo se calculóel coeficiente de correlación $r$ de Pearson entre estos valores y la media dada por las encuestas a cada fotografía. En este caso el índice de correlación hallado es $\mathrm{r}=0,94$; la pendiente de la recta ajustada $(b=1,0466)$ y la ordenada en el origen $(\mathrm{a}=-4,326)$ no se aproximan tanto a la ideal. (Gráfico 2). El tiempo empleado en el aprendizaje del método fue, por término medio, 10 horas.

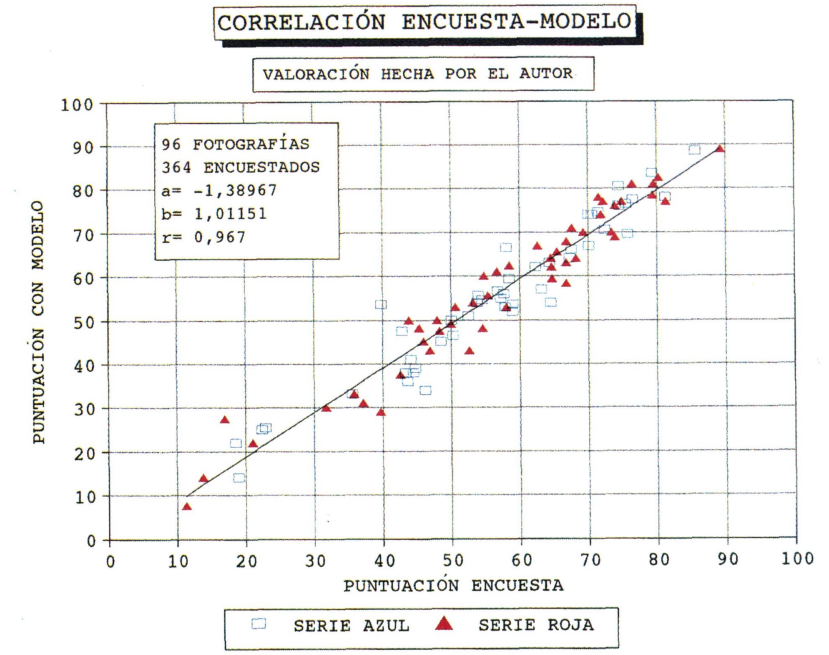

Gráfico 1.- Correlación entre las puntuaciones obtenidas por cada fotografia en la encuesta al público y la otorgada por los autores, valorándolas mediante el método propuesto.

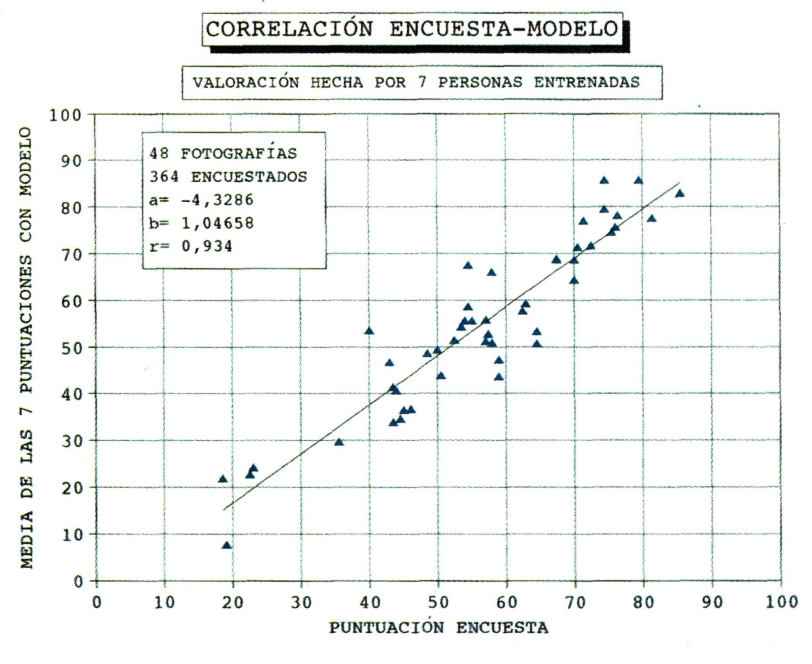

Gráfico 2.- Correlación entre las puntuaciones obtenidas por cada fotografia en la encuesta al público y la media de las otorgadas por siete personas entrenadas, valorándolas mediante el método propuesto.

Por lo que respecta a la capacidad del método para predecir el impacto originado por las construcciones en el paisaje, se pasa a continuación a describir una serie de ejemplos de aplicación, en los que se puede observar la estimación de este efecto que es posible obtener con la metodología propuesta.

\section{Modo de utilización del método}

La rutina a seguir es la siguiente: observando detenidamente el paisaje, se deben cumplimentar las dos hojas de la Ficha 2 (Modelo 1), que contienen los 16 descriptores, y tras esto traducir a valores cada una de las características 
apreciadas. Estos valores se reflejan en la Ficha 3 (Modelo 3), que nos permite ver rápidamente la puntuación obtenida por el paisaje en cada uno de los apartados.

Una vez valorado, para determinar el impacto paisajístico producido por la construcción en cuestión, basta con corregir los descriptores negativos, anulando aquéllos cuyo efecto desaparece y dando su valor correspondiente a los que pueden ser positivos. Una vez hecho esto, la evaluación del impacto viene directamente proporcionada por la diferencia de puntuación entre ambas valoraciones, corregida y sin corregir.

Se muestran a continuación dos casos de estimación del impacto visual producido por sendas instalaciones ganaderas, las que aparecen en las Fotografías $N^{\text {os }} 1$ y 2 , cuyos paisajes aparecen valorados en las fichas de los Ejemplos $\mathrm{N}^{\text {os }} 1$ y 2 respectivamente.

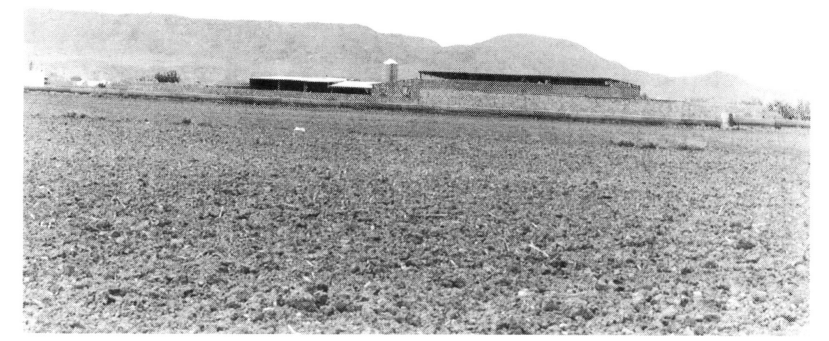

Foto 1.- Instalación ganadera del Ejemplo $N^{o} 1$. Su impacto visual se estimó en 6 puntos, sobre un paisaje valorado con 31 puntos.

\begin{tabular}{|c|c|c|c|c|c|}
\hline INUENTARIO & RECUF & Rsos P & ISAJE & & FICHA 2 \\
\hline \\
\hline \multicolumn{6}{|c|}{$\begin{array}{l}\text { MUNICIPIO: EJEMPLO FOTOGRAFIA NQ } 1 \\
\text { LOCALIZACIÓN (INSTALACIÓN): INSTALACIÓN GANADERA }\end{array}$} \\
\hline \multicolumn{6}{|c|}{$\begin{array}{l}\text { PTO DB OBSBRVACION : } \\
\text { NE: }\end{array}$} \\
\hline \multicolumn{6}{|c|}{ RECURSOS VISUALES } \\
\hline \multicolumn{6}{|l|}{$\nabla 1-A G O A$} \\
\hline A-IIPO & 2ONA PANTA & ARROYO & RIO & LAGO/PANTA & MAR \\
\hline B-QRILLAS & SIN VEGET & & CON VEGET. & & MUCH. VEG. \\
\hline C-Movin. & NINGUNO & LIGERO & MEANDROS & RAPIDO & CASCADA \\
\hline D-CANTIDAD & BAJA & & MEDIA & & A:TA \\
\hline \multicolumn{6}{|c|}{$\searrow 2$ 2-FORMA DBL TBRRBNO } \\
\hline (A) IIPQ & LLANO & $\cos 2 A$ & COLINAS & MONTAMOSO & \\
\hline \multicolumn{6}{|c|}{ X 3-VBGBTACIÓN } \\
\hline (A) CUBBERTA & (45) & $5-251$ & $25-50$ & $50-751$ & $275-100$ \\
\hline B-DIVERSID. & POCA & & PRESENTE & & BASTANTE \\
\hline C-CALIDAD & REGULAR & & BUENA & & MUY BUENA \\
\hline$D-\underline{T I P Q}$ & H. SECANO & B. REGADIO & ARBUSTIVO & PRADERA & ARBOREO \\
\hline \multicolumn{6}{|l|}{ Q 4-NIBVB } \\
\hline A-CUBIERTA & $<5$ & $5-25$ & $25-501$ & $50-751$ & $>75-100$ \\
\hline \multicolumn{6}{|l|}{$\triangle 5$ SADNA } \\
\hline A-PRESENCIA & PRESENTES & & & & ABUNDNNTB \\
\hline B-INTERÉS & MEDIOCRE & & & & BUENO \\
\hline C-FACILIDAD VERSE & MEDIOCRE & & & & BUENA \\
\hline \multicolumn{6}{|c|}{$\checkmark 6-\nabla S O S$ DBL SOBLO } \\
\hline (A) TIPQ. & INDUSTRIAL & MINERO & URBANO & AGRICOL. & SALVAJE \\
\hline B. INTENSIDAD POB & ROY POB. & & POBLADO & & POCO POBL. \\
\hline \multicolumn{6}{|l|}{ অ7-VISTAS } \\
\hline (A) MMPLITUD & $<450$ & $458-90$ & $908-1808$ & $1808-2708$ & 22700 \\
\hline (B) IIPQ & BAJA & & MEDIA & & PANORXYIICA \\
\hline \multicolumn{6}{|l|}{$\triangle 8$-SONIDOS } \\
\hline A-PRESENCIA & PRESENTES & & & & DOMINANTES \\
\hline$B-\underline{T I P Q}$ & MOLESTOS & & INDIFERENTE & & ARMONIOSOS \\
\hline \multicolumn{6}{|l|}{$\triangle 9$ 9-OLORES } \\
\hline A-PRESENCIA & PRESENTES & & & & DOMI NANTES \\
\hline$B-I I P Q$ & MOLESTOS & & INDI FERENTE & & ARMONIOSOS \\
\hline
\end{tabular}

Ejemplo $N^{o}$ 1.- Descriptores 1 a 9 del Inventario de Recursos del Paisaje de la Fotografia 1 (Ficha 2. Hoja 1).

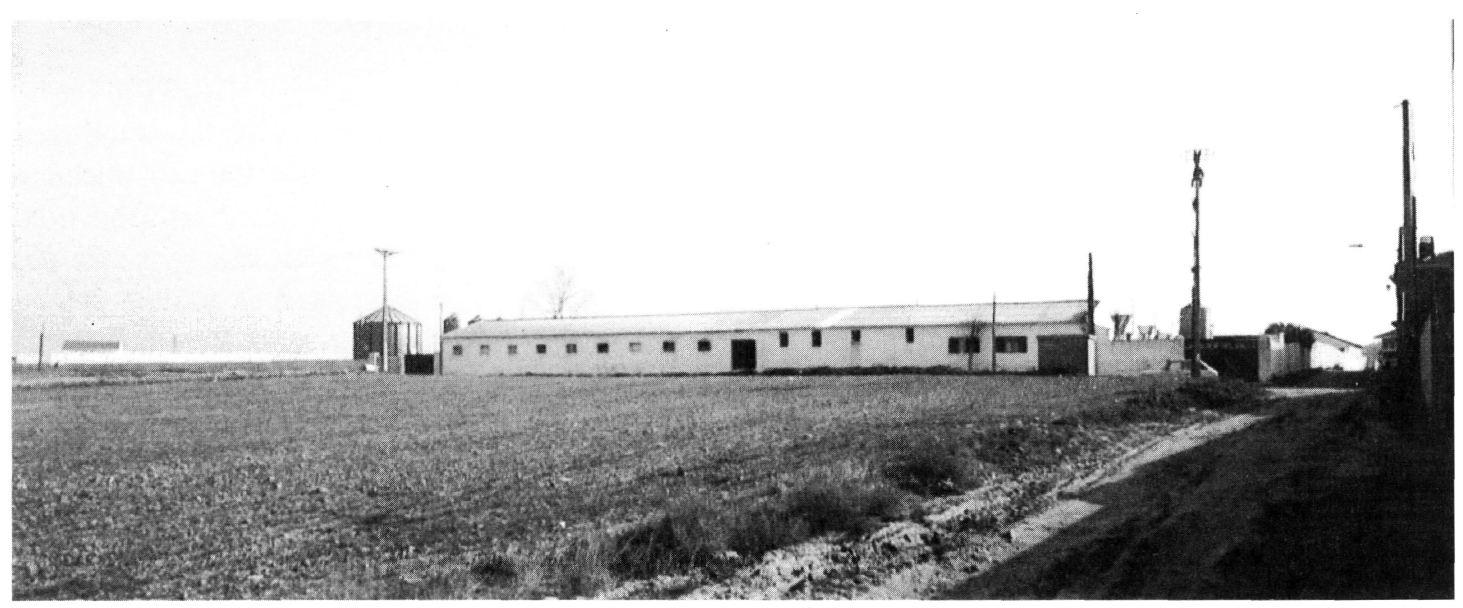

Foto 2.- Instalación ganadera del Ejemplo $N^{0} 1$. Su impacto visual se estimó en 6,5 puntos, sobre un paisaje valorado coń 26,5 puntos. 


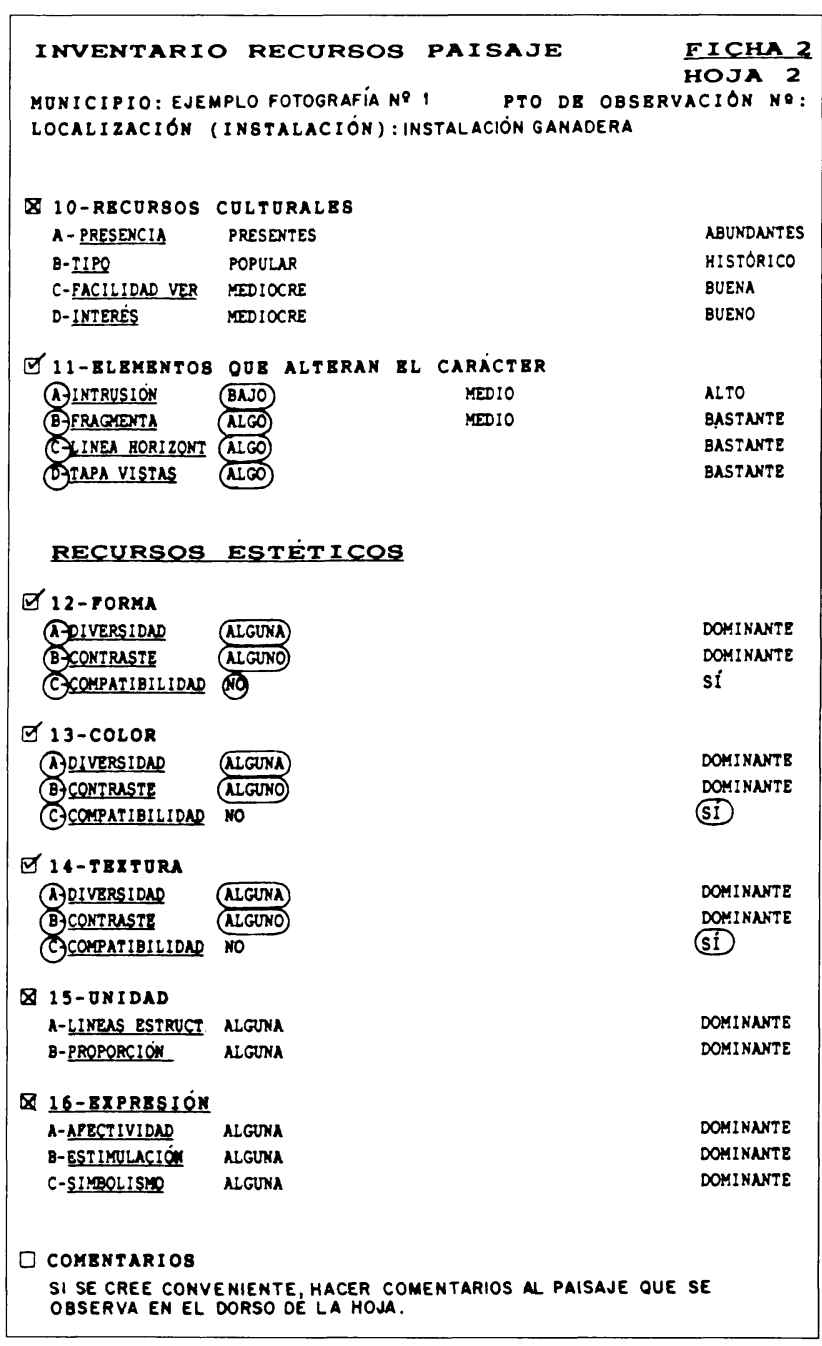

Ejemplo $N^{o}$ 1.- Descriptores 10 a 16 del Inventario de Recursos del Paisaje de la Fotografia 1 (Ficha 2. Hoja 2).

Como se puede apreciar, en el Ejemplo $N^{\circ} 1$, los puntos negativos se deben a los descriptores "Elementos que alteran el carácter" (-2) y "Forma" (-1). Si no estuviera la instalación, el primero ("Elementos que alteran el carácter") se anularía y el segundo ("Forma") tomaría el valor 3. Por este motivo, se puede estimar el impacto visual de esta edificación en 6 Puntos (31-25). Se concluiría que la existencia de esta instalación no ha motivado que el paisaje pertenezca a la categoría "Deficiente", pues sin ella no alcanzaría los puntos necesarios para ser clasificado como "Mediocre".

Sin embargo, la segunda instalación estudiada (Ejemplo $\mathrm{N}^{\circ}$ 2) si ha podido ser la responsable de que el paisaje pertenezca a la clase "Degradado", puesto que sin su presencia se habría incluido en la categoría "Deficiente". En este caso, los descriptores negativos son: "Olores" $(-1)$, "Elementos que alteran el carácter" $(-1,5)$ y "Forma" $(-1)$. Realizando las mismas operaciones que en

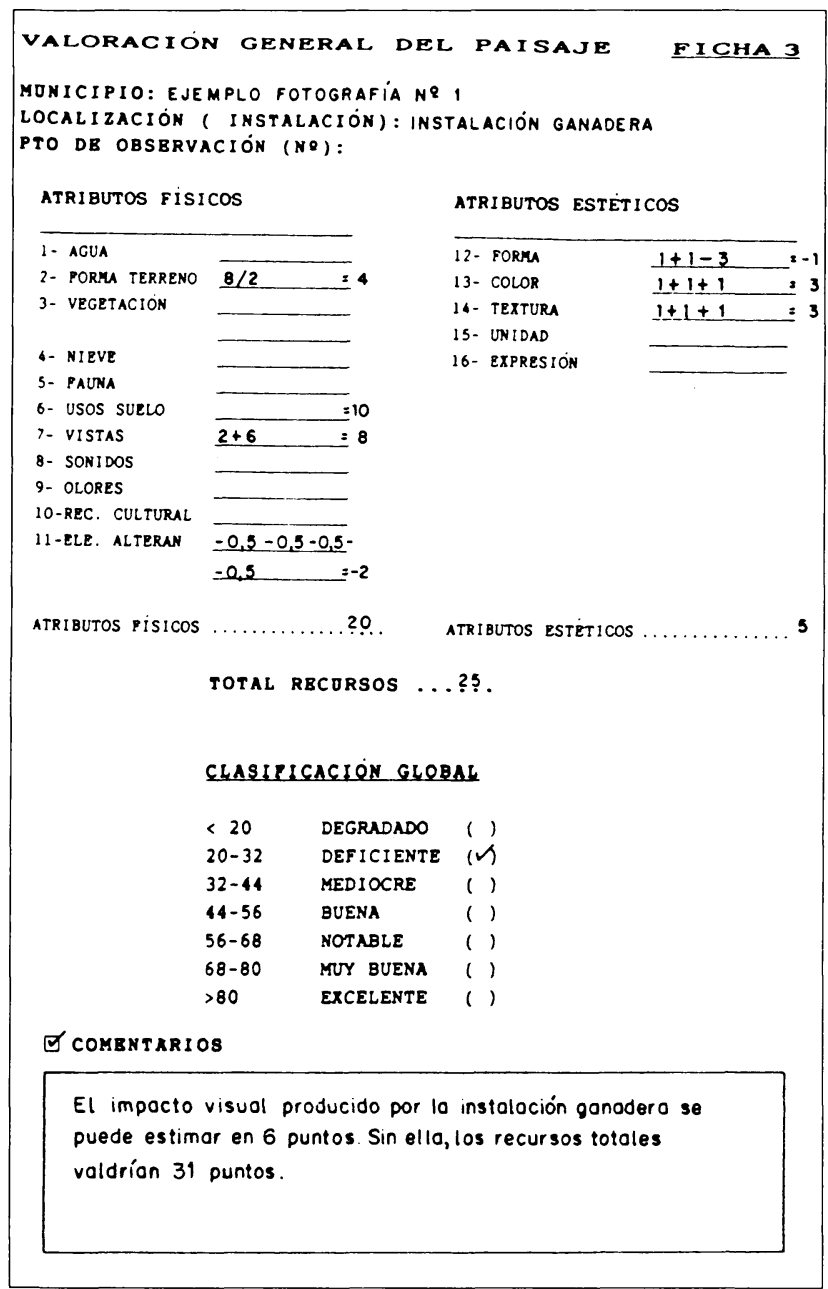

Ejemplo $N^{\circ} 1$-- Valoración General del Paisaje del Ejemplo $N^{\circ} 1$ (Ficha 3).

el caso anterior, obtendríamos que el impacto de esta construcción es de 6,5 Puntos (26,5-20), suficientes para motivar el descenso de categoría del paisaje.

\section{Algunos ejemplos de aplicación a otro tipos de construcciones}

Pese a estar pensado para evaluar el impacto paisajístico de las construcciones agrarias, el método podría ser aplicado en otros tipos de obras de ingeniería, puesto que durante el proceso de diseño se analizaron también varias metodologías no destinadas especificamente a aquellas (20) (21). Con el objeto de estudiar su comportamiento en este ámbito, seleccionamos aquellas fotografias evaluadas en las que aparecieran construcciones de otro tipo y corregimos su valoración. Una vez hecho esto, procedimos a comparar ambas fotografías (sin corregir y corregida), obteniendo los resultados que se muestran en la Tabla 3, cuyas fotos se comentan seguidamente. 


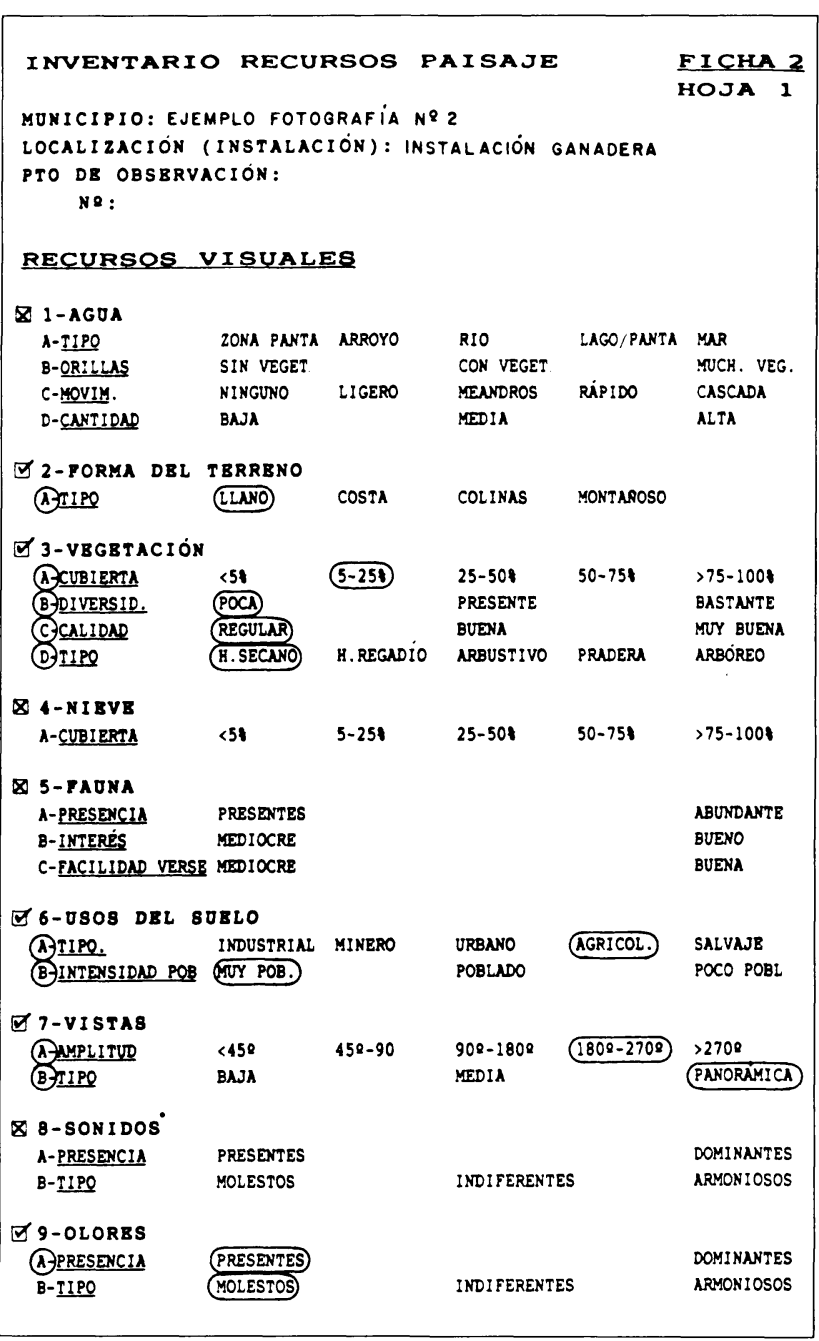

Ejemplo $N^{\circ}$ 2.- Descriptores 1 a 9 del Inventario de Recursos del Paisaje de la Fotografia 2 (Ficha 2. Hoja 1.).

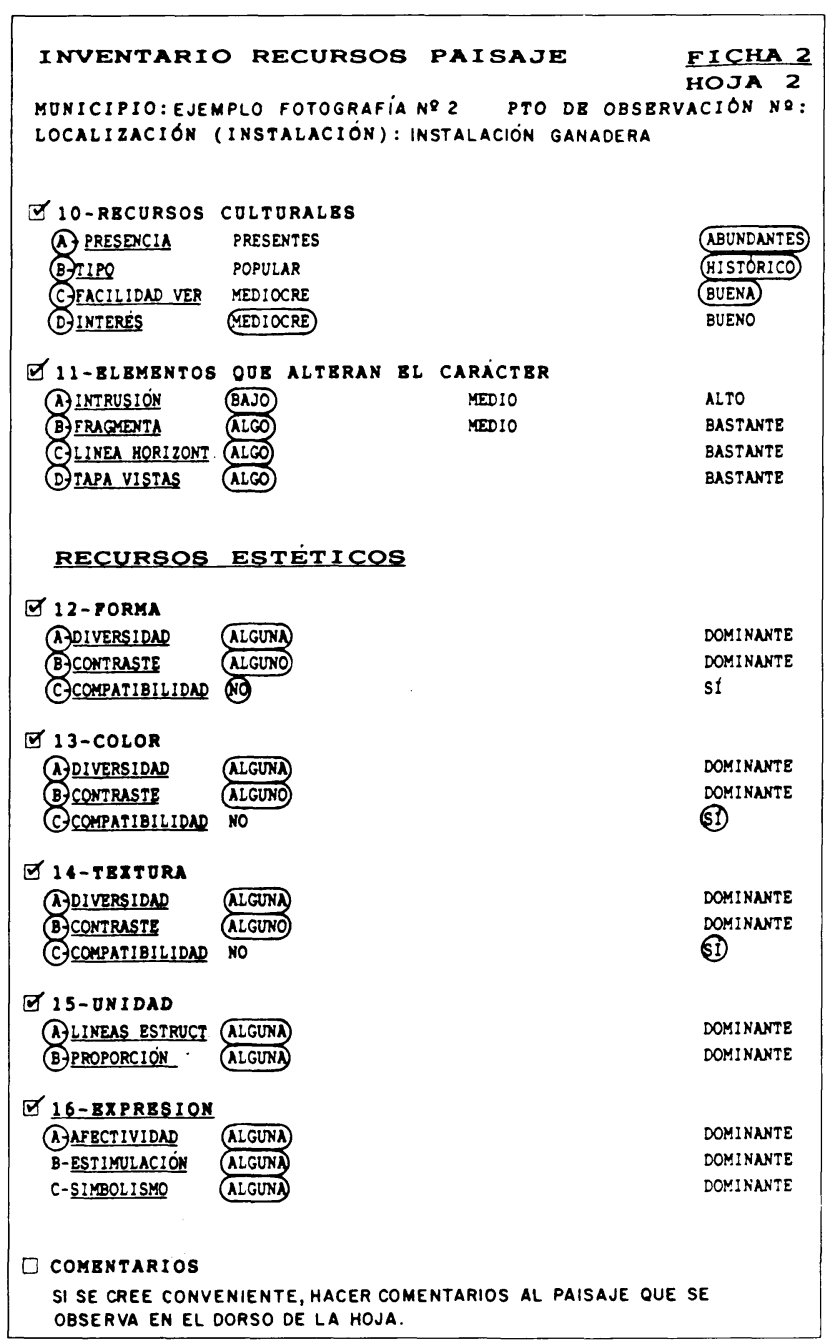

Ejemplo $N^{o}$ 2.- Descriptores 10 a 16 del Inventario de Recursos del Paisaje de la Fotografia 2 (Ficha 2. Hoja 2.).

TABLA 3

Cuantificación del impacto producido por las construcciones

\begin{tabular}{|c|c|c|c|c|c|}
\hline \multirow{2}{*}{$\begin{array}{l}\mathrm{N}^{\circ} \\
\text { FOTO }\end{array}$} & \multicolumn{2}{|c|}{ SIN CORREGIR } & \multicolumn{2}{|c|}{ CORREGIDAS } & \multirow{2}{*}{$\begin{array}{l}\text { VALOR } \\
\text { IMPACTO }\end{array}$} \\
\hline & VALOR & CATEGORÍA & VALOR & CATEGORÍA & \\
\hline 3 & 14 & Degradado & 28 & Deficiente & 14 \\
\hline 4 & 7,5 & Degradado & 20,5 & Deficiente & 13 \\
\hline 5 & 19,5 & Deficiente & 38,5 & Mediocre & 19 \\
\hline 6 & 33 & Mediocre & 40 & Mediocre & 7 \\
\hline 7 & 14 & Degradado & 41,5 & Mediocre & 27,5 \\
\hline
\end{tabular}




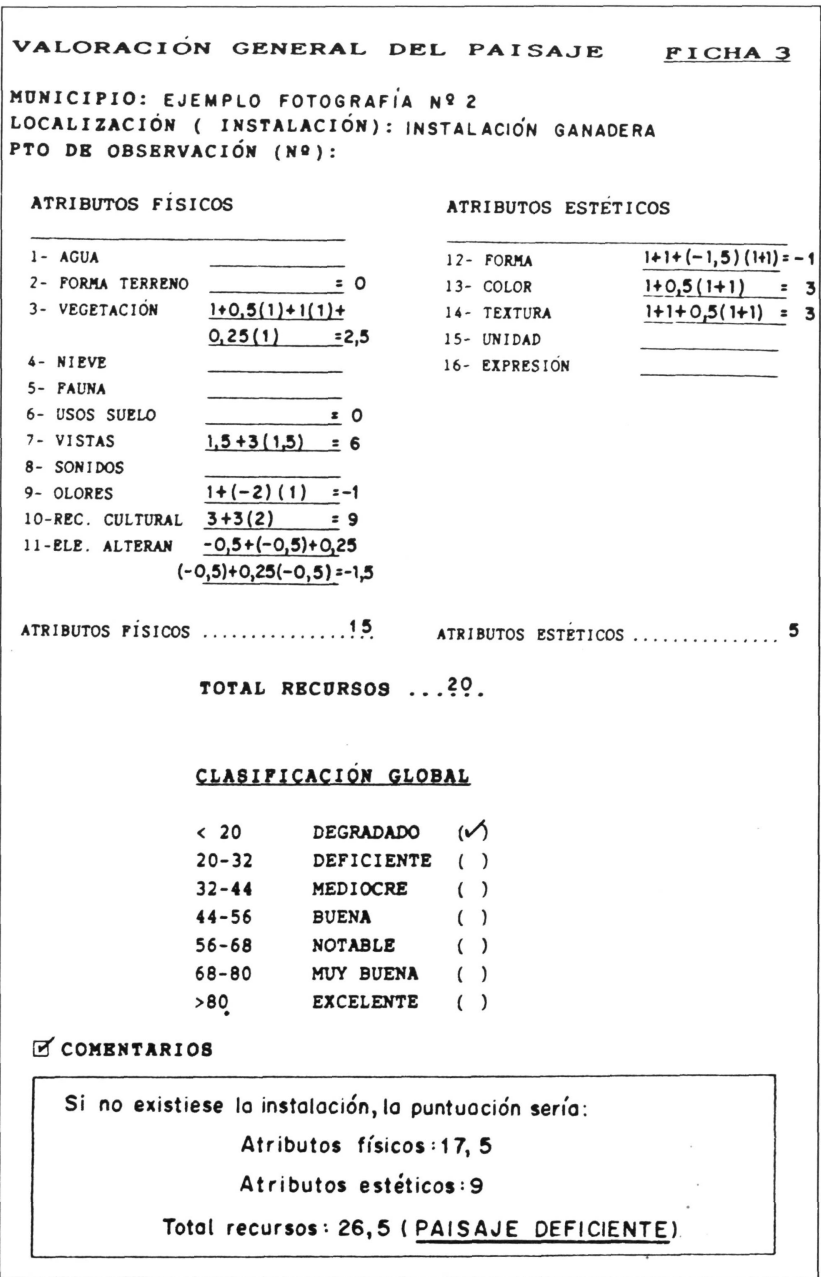

Ejemplo $N^{o}$ 2.- Valoración General del Paisaje del Ejemplo $N^{o} 2$ (Ficha 3)

\section{Fotografia 3}

Los descriptores que toman valores negativos son: "Elementos que alteran el carácter" $(-10)$ y Color $(-1)$. En el caso de no existir la instalación, estos descriptores tomarían los valores de 0 y 3 respectivamente. Con esta corrección, el impacto visual de la central se puede estimar en 14 puntos y el paisaje obtendría 28 puntos, pudiendo ser calificado como "Deficiente" en lugar de "Degradado".

\section{Fotografia 4}

El paisaje que muestrala fotografía, sin duda muy degradado, fue evaluado negativamente en los dos descriptores siguientes: "Elementos que alteran el caracter" (-9) y "Color" (-1). El nuevo valor que tomaría el descriptor "Color" sería de 3 puntos, con lo que el impacto de la planta sería de 13 puntos, pasando el paisaje de "Degradado" (7,5 Puntos) a "Deficiente" (20,5 Puntos).

\section{Fotografia 5}

El "scalextric" de la imagen solamente fue evaluado negativamente en los descriptores "Elementos que alteran

(c) Consejo Superior de Investigaciones Científicas Licencia Creative Commons 3.0 España (by-nc) el carácter" (-7 puntos) y "Forma" (-3). Este último, una vez corregido tomaría el valor de 9 , con lo que el impacto visual del enlace sería de 19 puntos. Se puede decir que tras la construcción de la carretera, el paisaje cambió de la categoría "Mediocre" (38,5 Puntos) a la "Deficiente".(19,5 Puntos).

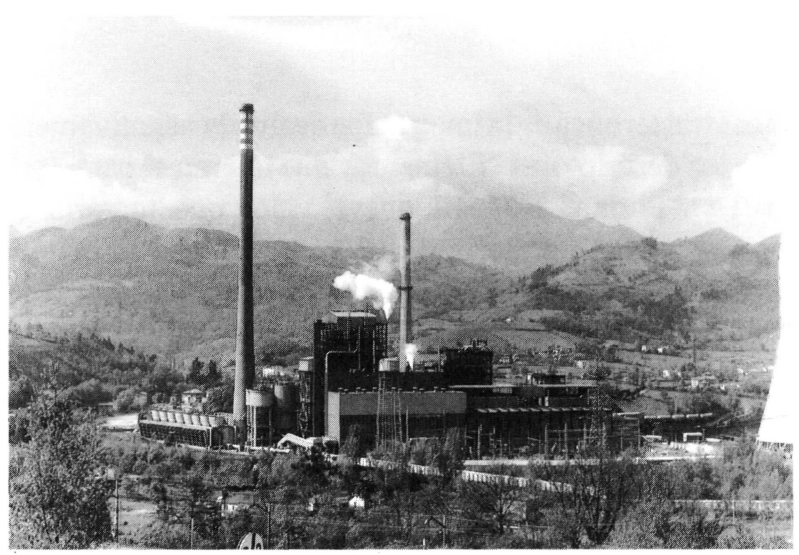

Foto 3.- Central cuyo impacto visual se estimó en 14 puntos, sobre un paisaje valorado con 28 puntos.

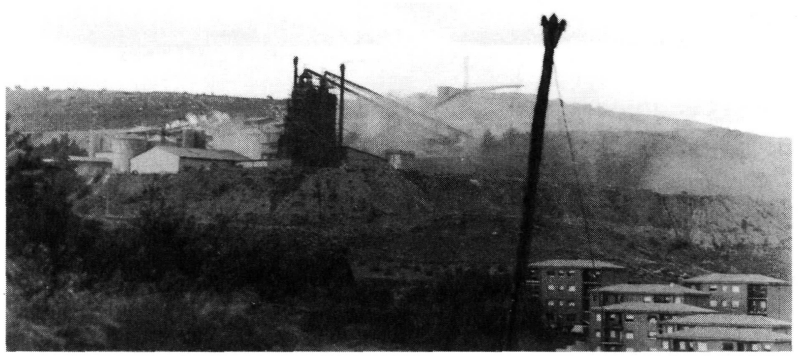

Foto 4.- Planta cuyo impacto visual se estimó en 13 puntos, sobre un paisaje valorado con 20,5 puntos.

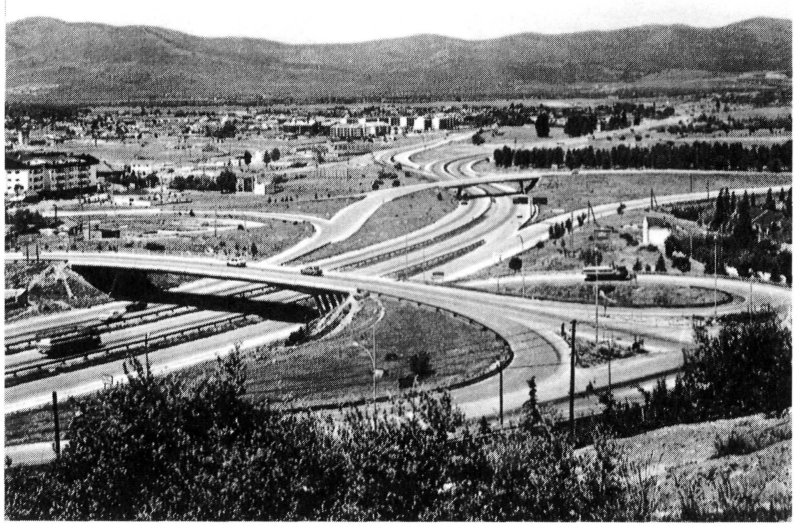

Foto 5.- "Scalextric" cuyo impacto visual se estimó en 19 puntos, sobre un paisaje valorado con 38,5 puntos. 


\section{Fotografia 6}

Este paisaje se penalizó con 1 punto negativo en concepto de "Elementos que alteran el carácter", y con 3 negativos en el descriptor "Color". El impacto de la edificación sería de 7 Puntos, pues el "Color" corregido alcanzaría 3 puntos.

\section{Fotografia 7}

Lacentral térmica de la imagen fue evaluada negativamente en los descriptores "Elementos que alteran el carácter" (-10) y "Forma" (-5). El nuevo valor que tomaría el descriptor "Forma" sería de 12,5 puntos, con lo que su impacto visual valdría 27,5 puntos, pasando el paisaje de "Degradado" (14 Puntos) a "Mediocre" (41,5 Puntos).

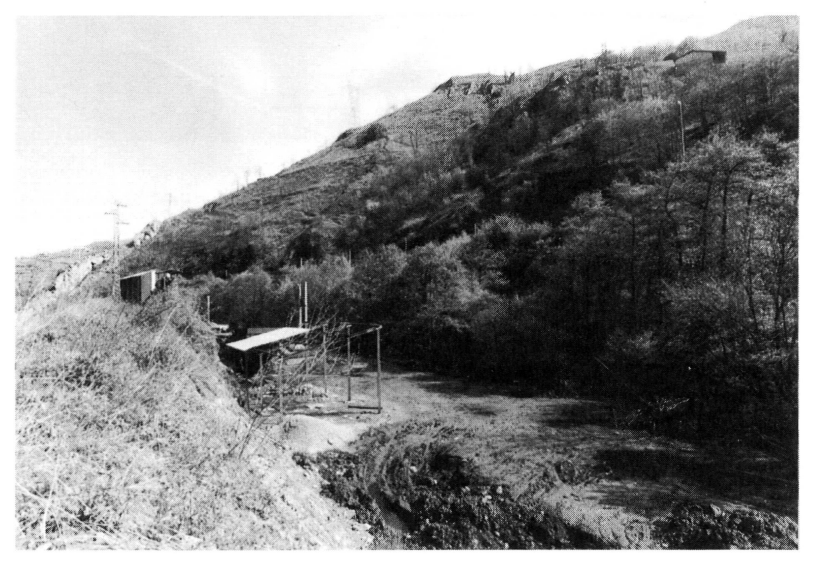

Foto 6.- Construcción cuyo impacto visual se estimó en 7 puntos, sobre un paisaje valorado con 40 puntos.

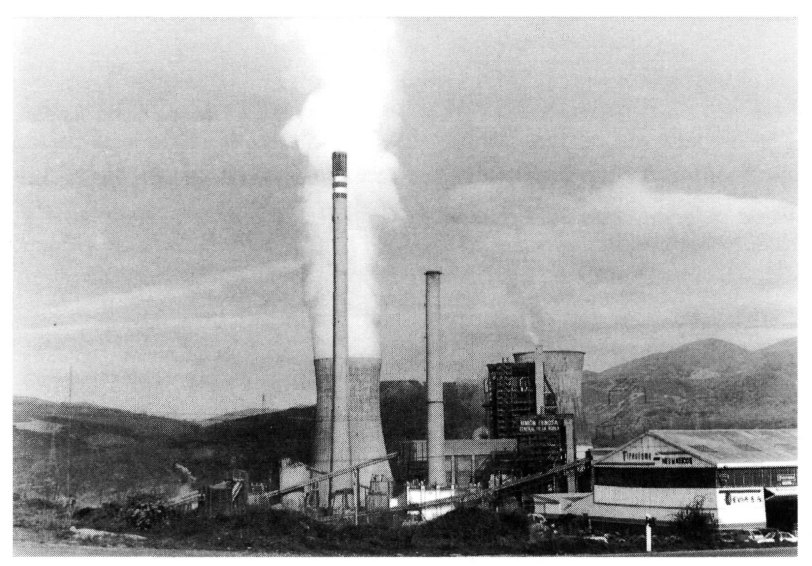

Foto 7.- Central cuyo impacto visual se estimó en 27,5 puntos, sobre un paisaje valorado con 401,5 puntos.

\section{Conclusiones}

1.- Tras la realización del ensayo, se ha visto cómo el método puede resultar muy válido para estimar la opinión del público sobre la calidad visual de un paisaje.
2.- Parece probable que los resultados obtenidos con la aplicación del método por parte de un técnico que haya invertido unas 10 horas en su estudio, serán muy similares a los obtenidos por los autores.

3.- Utilizando el método, parece posible llegar a cuantificar el impacto visual producido por las construcciones en el paisaje, tanto "ex ante" como "ex post".

\section{Agradecimientos}

Los autores quieren expresar su agradecimiento a la Comisión Interministerial de Ciencia y Tecnología del Ministerio de Educación y Ciencia y a la Xunta de Galicia, gracias a cuyas financiaciones se ha llevado a cabo este trabajo, como parte de los proyectos de investigación titulados "Estudio de los métodos de valoración de los recursos visuales en el ecosistema rural. Estimación del impacto producido por las nuevas construcciones. Caso particular de Castilla". (Proy. CICYT NAT 90-0099) y "Estimación del impacto visual de las construcciones agrarias en el paisaje rural gallego". (Proy. XUGA 29-102 A94).

\section{BIBLIOGRAFÍA}

(1) CAÑAS GUERRERO, I.; Integración de las construcciones agrarias en el paisaje: el color. Tesis doctoral. Universidad Politécnica de Madrid. (Sin publicar).

(2) DEARDEN, P.; Consensus and theoretical framework for landscape evaluation. Journal Environmental Management. $\mathrm{N}^{\circ} 34$, 1987. Págs. 267-268.

(3) CARLSON, A.A.; Formal qualities in the natural environment. Jorunal of Aesthetics Education. Vol. 13, 1979. Págs. 99-114.

(4) DANIEL, P. Y BOSTER, R.S.; Measuring landscape aesthetics: the scenic beauty simulation method. Research PaperRM167. USDAForest Service. RMFRES. Fort Collins, Colorado. 1976.

(5) RAMOS, A. ET AL.; Visual landscape evaluation, a gred technique. Landscape Planning. Vol. 3, 1976. Págs. 67-88.

(6) TETLOW, E. J. Y SHEPPARD, R. J.; Visual unit analysis: a descritptive aproach to landscape assesment. Proceedings of our national landscape. Abrit, 1979.

(7) LITTON, R.B.; Visual assesment of natural landscapes. Environmentlaaesthetics: Essays in interpretation. Western geographical series $\mathrm{N}^{\circ}$ 20. 1982, Págs. 97-115.

(8) ZUBE, E.H.; SELL, J.L. Y TAYLOR, J.G.; Landscape perception: research, application and theory. Landscape planning. $\mathrm{N}^{\circ} 9,1982$. Págs. 1-33. 
(9) GONZÁLEZBERNÁLDEZ, F. Y PARRA, F.; Dimensions of landscape preferences from pairwise comparation. Proceedings of our national landscape. Abril, 1979.

(10) BARRIOS, J.C. ET AL.; Content analysis of landscape preferences: the environmental perception of Madrid livestock raiser. Landscape research. 10(3), 1986. Págs. 2-8.

(11) BERNÁLDEZ, F. G. Y GALLARDO, D.; Environmental chalenge and environmental preference: age and sex efects. Journal of environmental chalenge. $\mathrm{N}^{\circ} 28,1989$. Págs. 5370 .

(12) PRICE, C.; Subjetivity and objetivity in landscape evaluation. Environment and planning. Vol. 3, 1976. Págs. 829-838.

(13) USDA Forest Service; National forest landscape management. Agriculture handbook number 462. U.S. Government Printing Office. Washington. 1974.

(14) USDA Soil Conservation Service; Procedures to establish priorities in landscape architecture. U.S. Government Printing Office. Washington. 1978

(15) USDI Bureau of Land Management; Visual resource management. Government Printing Office. Washington 1980.

(16) CAÑAS GUERRERO, I.; Las construcciones agrarias en la Comunidad de Madrid y sus condicionantes estéticos según las normas subsidiarias. Informes de la Construcción. Vol 45- $\mathrm{N}^{\circ} 429$, enero/febrero, 1994. Págs. 33-48.

(17) CAÑAS GUERRERO,I.; GARCÍA GARCÍA,A.I.; Principales variables que inciden en la integración de las construcciones agrarias en el paisaje. Informes de la Construcción. Vol. 46- $\mathrm{N}^{\circ}$ 433, septiembre/octubre, 1994 Págs. 47-55.

(18) CAÑASGUERRERO,I.; Introducción al paisaje. $1^{\text {a }}$ edición. Unicopia, Lugo. 1995.

(19) CAÑAS GUERRERO,I.; Valoración del paisaje. $1^{\mathrm{a}}$ edición Unicopia, Lugo. 1995.

(20) USDOT; Visual impact assessment for highway projets Federal Highway Administration Office of Environmental Policy. Washington. 1981.

(21) CAÑAS GUERRERO,I.; Estimación del impacto paisajístico de las carreteras. Informes de la Construcción. Vol. 45- $\mathrm{N}^{\mathrm{o}}$ 425-426, mayo/junio-julio/agosto,1993. Págs. 43-54

\section{Publicación del IETCC/CSIC}

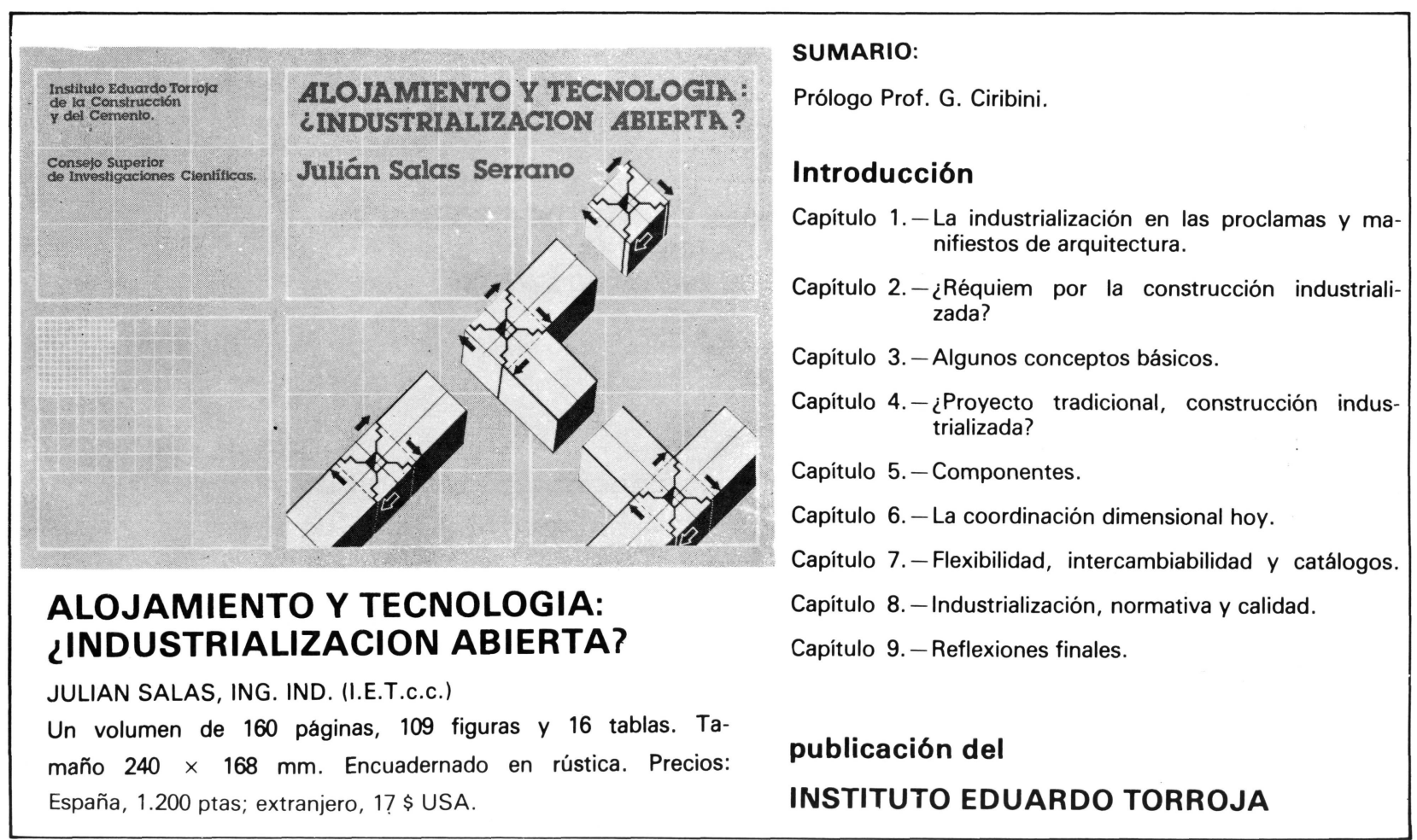

(c) Consejo Superior de Investigaciones Científicas Licencia Creative Commons 3.0 España (by-nc) 